\title{
Analysis of Induction Motor Cascading Stall in a Simple System Based on the CASCADE Model
}

\author{
Hao Wu, Member IEEE and Ian Dobson, Fellow IEEE
}

\begin{abstract}
It is common for many induction motors to be connected to a load bus, and voltage can sometimes quickly collapse when an initial disturbance triggers a cascade of motors stalling. We analyze a single line power system supplying a bus with many induction motors. Variation and uncertainty in the motor load is modeled probabilistically. We describe an analytic method to estimate the parameters of a model of cascading failure from system parameters, and hence estimate the probabilities of the number of motors stalled. The analytic method gives insights into cascading stall and relates system parameters to the risk of cascading stall.
\end{abstract}

Index Terms-induction motor, cascading failure, load modeling, voltage stability.

\section{NOMENCLATURE}

\begin{tabular}{|c|c|}
\hline$V$ & Load bus voltage \\
\hline$P_{L}, Q_{L}$ & Real and reactive power for total load \\
\hline$G_{Z}, B_{Z}$ & $\begin{array}{l}\text { Constant conductance and susceptance of } \\
\text { arallel load }\end{array}$ \\
\hline$N$ & Number of motors connected to load bus \\
\hline$s$ & Slip of motor \\
\hline subscript 0 & Indicates quantity at initial state, e.g., $s_{0}$ \\
\hline subscript $i$ & Indicates quantity for motor $i$, e.g., $s_{i}$ \\
\hline$C_{i}$ & $\begin{array}{l}\text { Factor converting equivalent admittance for } \\
\text { motor } i \text { from motor base to system base }\end{array}$ \\
\hline$Y_{M}, G_{M}, B_{M}$ & $\begin{array}{l}\text { Admittance, conductance and susceptance } \\
\text { of all motors }\end{array}$ \\
\hline $\begin{array}{l}\text { subscript } M \\
\text { for } Y\end{array}$ & $\begin{array}{l}\text { Motor admittance including compensator } \\
\text { at system base }\end{array}$ \\
\hline $\begin{array}{l}\text { subscript } M T \\
\text { for } Y\end{array}$ & $\begin{array}{l}\text { Motor admittance of T-shaped equivalent } \\
\text { circuit at motor base }\end{array}$ \\
\hline $\begin{array}{l}\text { superscript } n \\
\text { and } s \text { for } Y\end{array}$ & $\begin{array}{l}\text { Admittances of motor at normal state and } \\
\text { stalled state respectively }\end{array}$ \\
\hline$M_{\text {ratio }}$ & $\begin{array}{l}\text { Ratio between initial total motor power and } \\
\text { initial total load power }\end{array}$ \\
\hline$P_{\text {ratio }}$ & $\begin{array}{l}\text { Ratio of the maximum motor initial power } \\
\text { to the minimum motor initial power }\end{array}$ \\
\hline$V_{c r}$ & Critical voltage for motor \\
\hline$V_{\min }, V_{\max }$ & $\begin{array}{l}\text { Lower and upper limits of distribution of } \\
V_{c r}\end{array}$ \\
\hline$N_{s}$ & Number of motors stalled at steady-state \\
\hline$V_{s}$ & Load bus voltage at steady-state \\
\hline$V_{\text {post }}$ & Load bus voltage after initial disturbance \\
\hline
\end{tabular}

Hao Wu is with the College of Electrical Engineering, Zhejiang University, Hangzhou, Zhejiang, China, 310027. vuhao@zju.edu.cn. Ian Dobson is with the Electrical and Computer Engineering Department, Iowa State University, Ames IA 50011 USA. dobson@iastate.edu. Financial support in part from China NSF grant 50707029 (Wu), and USA DOE grant DE-SC0002283 and NSF grant CPS-1135825 (Dobson) is gratefully acknowledged. CIEEE 2013
$\bar{Y}_{M} \quad$ Expected admittance of all motors

$\bar{N}_{s} \quad$ Expected number of motors stalled at steady-state

$\bar{V}_{s} \quad$ Expected load bus voltage at steady-state

$\bar{V}_{D} \quad$ Expected voltage drop after initial disturbance

$\bar{V}_{P} \quad$ Expected voltage drop when an extra motor stalled

$d, p \quad$ Size of initial disturbance and the amount of propagation in CASCADE model

\section{INTRODUCTION}

Induction motors are one of the most important dynamic loads in power systems and have significant impacts on power system dynamic performance and stability [1], [2], [3], [4], [5], [6]. Decades of effort have improved the modeling of induction motor loads [7], [8], [9], [10], [11], [12], so that the power system can be analyzed more accurately and hence operated more securely and economically. Although great progress has been achieved, it remains one of the most demanding research topics in power system modeling and analysis.

The characteristics of time variation, dispersed location, operational discontinuity, parameter uncertainty, and diversity of type make load modeling a difficult task. Early works usually try to use an equivalent load with one induction motor and a static load component to comprise all these characteristics. In recent years, researchers have begun to investigate specific load characteristics in detail; for example, [13] studies the discontinuous characteristic of motor stalling using bifurcation theory, [14] studies the statistical characteristic of induction motor models under parametric uncertainties using Monte Carlo simulation, [15] studies the effect of location and motor parameter on system eigenvalues using parametric sensitivity, [12] addresses the characteristics of type diversity with a load model representing an air conditioner compressor and its protection logic. However, modeling several motor characteristics simultaneously remains a challenge.

Cascading failure of power transmission systems has become a research focus in the last decade [16], [17], [18]. While far from mature, it has already provided some new perspectives and modeling approaches, some of which can potentially apply to other forms of cascading failure. Among them, the CASCADE model is a high-level and generic probabilistic model for cascading failure [19]. The CASCADE model provides a simple formula to predict the probability of number of components failed in terms of parameters that describe the size of the initial disturbance and the average amount of propagation of failures.

In [20], we introduce a new probabilistic model of cascading stall of many induction motors attached to a load bus and supplied by a simple power system equivalent. Voltage drop due to induction motor stall is a cascading process in which motor stall tends to reduce bus voltages and make it more 


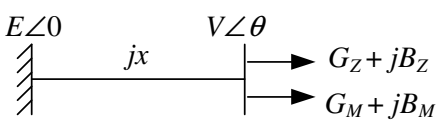

Fig. 1. Single load infinite bus system

likely that further motors stall. The quasistatic modeling and simulation of cascading stall is discussed and justified. In particular, the variation and uncertainty in a load comprising the hundreds of induction motors that arises in practice is represented in [20] by probabilistic parameters for the induction motors. The outcome of disturbances to the power system is also probabilistic, and the distribution of the number of motors stalled is simulated. This approach can reveal the risk of occasional events in which large numbers of motors stall. We found that the simulated results of cascading stall could be fit in many cases by the CASCADE model, suggesting an intriguing commonality between cascading motor stall and other cascading processes.

We adopt the same system and modeling assumptions as [20], but instead of simulating and then fitting the CASCADE model to merely summarize the simulation results as is done in [20], in this paper we analyze the system to derive the CASCADE model parameters from the power system and induction motor parameters, and hence analytically predict the distribution of the number of stalled motors. The objectives are to establish the use of the CASCADE model and its parameters to describe cascading stall and to understand how system parameters influence the risk of cascading stall. We note the value of physics-based analysis of a simple system that reveals and quantifies basic relationships that can inform the understanding and engineering of the more complicated systems encountered in practice.

The paper is organized as follows. Section II reviews the system modeling and section III reviews the CASCADE model. Section IV does the analysis, section V presents the case studies, and section VI concludes the paper.

\section{Modeling AND ASSUmptions}

This section summarizes the modeling and assumptions established in [20]; see [20] for details.

\section{A. System modeling}

Fig. 1 shows a single load infinite bus system, where the load comprises $N$ induction motors and a constant impedance load. $E \angle 0$ is the infinite bus voltage, $V \angle \theta$ is the load bus voltage, $x$ is the transmission line reactance, $G_{Z}+j B_{Z}=Y_{Z}$ is the admittance for constant impedance load, and $G_{M}+$ $j B_{M}=Y_{M}$ is the equivalent admittance for $N$ motors.

At any moment of the underlying dynamic process, $Y_{M}$ can be regarded as a constant, and hence $V$ can be obtained by

$$
V=\frac{E}{x \sqrt{\left(G_{Z}+G_{M}\right)^{2}+\left(B_{Z}+B_{M}-\frac{1}{x}\right)^{2}}} .
$$

The motors use a T-shaped equivalent circuit [21] as shown in Fig. 2, where $s$ is the motor slip, and $r_{1}, x_{1}, r_{2}, x_{2}, r_{m}$

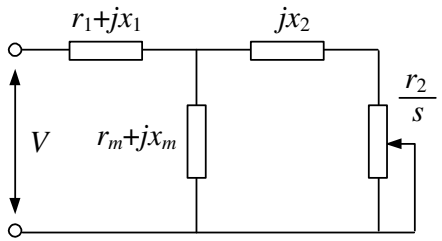

Fig. 2. T-shaped equivalent circuit for the induction motor

and $x_{m}$ are the resistances and inductances for static, rotor and magnetic windings respectively. The electromagnetic torque is

$$
T_{e}(s, V)=\frac{r_{2}}{s}\left|\frac{z_{m}}{z_{1} z_{m}+\left(z_{m}+z_{1}\right) z_{2}(s)}\right|^{2} V^{2}
$$

and the mechanical torque is

$$
T_{m}(s)=k\left[\alpha+(1-\alpha)(1-s)^{p}\right],
$$

where $z_{1}=r_{1}+j x_{1}, z_{2}(s)=r_{2} / s+j x_{2}, z_{m}=r_{m}+j x_{m}$, $k$ is the load factor, $\alpha$ is the ratio of constant mechanical torque to total torque, and $p$ is a factor reflecting the impact of motor rotation speed on the non-constant mechanical torque component.

\section{B. Assumptions and formulation}

For the system, it is assumed that $V$ drops in a quasistatic and monotonic manner from the initial load bus voltage $V_{0}$. For the $N$ motors, it is assumed that 1) they have same parameters, excluding initial operation conditions; 2) each motor's critical voltage $V_{c r}$, below which the motor stalls, is uniformly distributed ${ }^{1}$ in the interval $\left.\left[V_{\min }, V_{\max }\right] ; 3\right)$ the ratio of any two motors' initial power is not greater than $P_{\text {ratio }}$, and total initial motor power is allocated to each motor according to a weight uniformly distributed in $\left[1, P_{\text {ratio }}\right]$.

Based on the quasistatic assumption, the motors can be described by a static torque balance equation $T_{e}(s, V)=T_{m}(s)$, which can be rewritten as

$$
\frac{V^{2}}{k}=h(s),
$$

where

$$
h(s)=\left[\alpha+(1-\alpha)(1-s)^{p}\right] \frac{s}{r_{2}}\left|z_{1}+\left(1+\frac{z_{1}}{z_{m}}\right) z_{2}(s)\right|^{2} .
$$

Combined with the quasistatic system assumption, the second assumption for motors implies that the motor is stable if and only if its $V_{c r}$ is less than $V$, otherwise it is stalled. The existence of $V_{c r}$ indicates that $h(s)$ in (2) has a local minimum in the vicinity of initial slip $s_{0}$. Denote the $s$ at which $h(s)$ is minimized as $s_{c r}$. From (2) and the first assumption for motors, we know that $s_{c r}$ and hence $V_{c r}^{2} / k$ are inherent constants of the motors.

The motor initialization procedures are as follows: Given $V_{c r i}$ for motor $i, k_{i}$ is determined from (2) at the critical point. Then, given $V_{0}, s_{0 i}$ is determined from (2) evaluated at the initial point. With $s_{0 i}$, the compensator $B_{c i}$ can be obtained, which ensures that the power factor of the motor and the initial

\footnotetext{
${ }^{1}$ Lefèvre [23] discusses generalizations of the CASCADE model that relax the assumption of a uniform distribution of $V_{c r}$.
} 
load power $P_{L 0}+j Q_{L 0}$ are consistent. Finally, the factor $C_{i}$ that converts admittance in motor base to the system base can be calculated. Once $k_{i}, B_{c i}$, and $C_{i}$ are initialized, they are kept constant during the cascading process. Moreover, from the above procedures, it can be seen that they are all functions of $V_{c r i}$ and that the distribution of initial motor power determines $C_{i}$.

No matter what the underlying dynamic process is, at steady state, the motor can be regarded as having only two states: stalled and normal. From the equivalent circuit, the motor admittances at stalled and normal states are

and

$$
Y_{M T}^{s}=\frac{z_{2}+z_{m}}{z_{1} z_{2}+z_{1} z_{m}+z_{2} z_{m}}
$$

$$
Y_{M T}^{n}\left(s_{i}\right)=\frac{z_{m}+z_{2}\left(s_{i}\right)}{z_{1} z_{m}+\left(z_{1}+z_{m}\right) z_{2}\left(s_{i}\right)}
$$

respectively. From (2), it can be seen that $s_{i}$ is a function of $V$ and $V_{c r i}$. Synthesizing all of the above, the admittance of motor $i$ in system base is

$$
Y_{M i}= \begin{cases}Y_{M i}^{n}\left(V, V_{c r i}\right)=C_{i}\left(Y_{M T i}^{n}+j B_{c i}\right) & \text { if } V_{c r i} \leq V \\ Y_{M i}^{s}\left(V_{c r i}\right)=C_{i}\left(Y_{M T i}^{s}+j B_{c i}\right) & \text { if } V_{c r i}>V .\end{cases}
$$

The admittance of the total motor load is then

$$
Y_{M}(V)=\sum_{i=1}^{N} Y_{M i}
$$

which is a function of $V$ and the $N$ motors' $V_{c r i}$ and the initial powers.

The solution of (1) and (4) gives the post-fault steady state voltage $V_{s}$. Algorithmic details and more explanations about system modeling and assumptions are omitted here due to space limitations and are given in [20].

\section{CASCADE MODEL}

Here we summarize the CASCADE model; for a detailed explanation see [22] and [19]. CASCADE is an idealized model of cascading failure that predicts the number of failed components of $N$ identical components with a simple analytic formula [19]. The model assumes each component has a random initial load in the interval $\left[L^{\min }, L^{\max }\right]$ and fails when load $L$ exceeds $L^{\text {fail }}$. After a component fails, a fixed and positive amount of load $P$ is transferred to each of the components, which may cause further failures in a cascade. To start the cascade, an initial disturbing load $D$ is applied to each component.

Fig. 3 illustrates the CASCADE model, where $L^{1}, \ldots, L^{N}$ are loads for $N$ components respectively, $X_{0}$ is the number of components failed after the initial disturbance, and $X_{1}$ and $X_{2}$ are the number of components failed at stage 1 and stage 2 of the cascade respectively.

With the normalizations

$$
d=\frac{D+L^{\max }-L^{\text {fail }}}{L^{\max }-L^{\min }} \text { and } p=\frac{P}{L^{\max }-L^{\min }},
$$

the five parameters of CASCADE model are reduced to two key parameters $d$ and $p$. $d$ describes the size of the initial disturbance, and $p$ describes the amount of propagation of

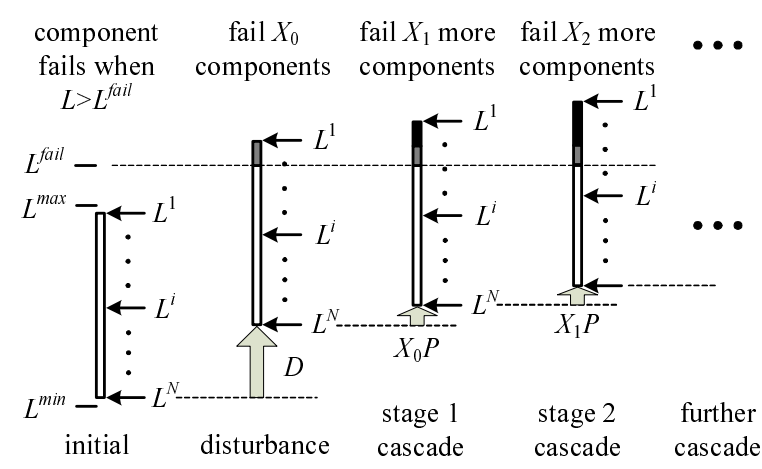

Fig. 3. An illustration of the CASCADE model

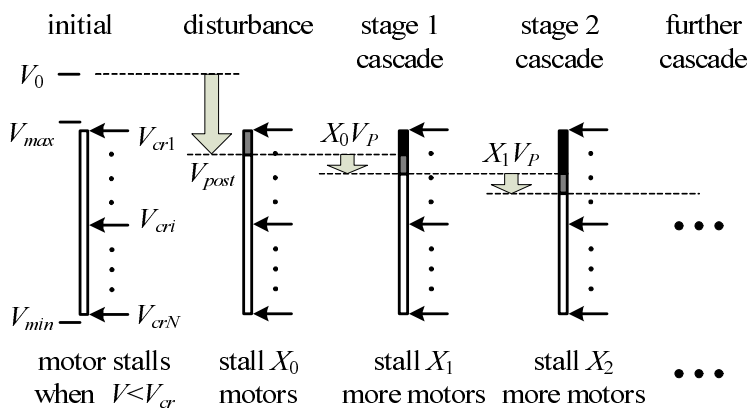

Fig. 4. Conceptual illustration of induction motor cascading stall

failures [19]. There is no cascading for $d<0$, and nontrivial cascades become possible for $d \geq 0$. For $d \geq 0$, the probability distribution of the number of failed components can be expressed in the simple formula

Probability of $r$ total failures $=$

$$
\begin{cases}\left(\begin{array}{c}
N \\
r
\end{array}\right) d(r p+d)^{r-1}(\max \{1-r p-d, 0\})^{N-r} ; & r<N, \\
1-\sum_{n=0}^{N-1} \text { probability of } n \text { total failures; } & r=N .\end{cases}
$$

\section{ANALYSIS OF INDUCTION MOTOR CASCADING STALL}

\section{A. Outline of the analysis}

A conceptual illustration of induction motor cascading stall is shown in Fig. 4, where $V_{\text {post }}$ and $X_{0}$ are the load bus voltage and the number of motors stalled after initial disturbance respectively, $V_{P}$ is the voltage drop after each motor stalled, and $X_{1}$ and $X_{2}$ are the number of motors stalled at stages 1 and 2 of the cascading stall process respectively. Comparing with Fig. 3, we see that the illustrative concept of motor cascading stall in Fig. 4 shares the same form as CASCADE. The difference that motors in Fig. 4 have fixed loads and a dropping failure threshold, whereas components in Fig. 3 have gradually increasing loads and a fixed failure threshold, is a superficial difference that does not affect the cascading process. It can be seen that $V_{\min }, V_{\max }, V_{P}$, and $V_{\max }-V_{\text {post }}$ play the same roles as $L^{\min }, L^{\max }, P$, and $D+L^{\max }-L^{\text {fail }}$ respectively. Define

$$
V_{D}=V_{\text {max }}-V_{\text {post }}
$$


which stands for the value of voltage lower than $V_{\max }$ after the initial disturbance. We will exploit this correspondence to estimate the CASCADE parameters $d$ and $p$ that describe the cascading stall of induction motors, and hence estimate the probability distribution of the number of stalled motors.

Although the conceptual model of induction motor cascading stall fits the CASCADE model well, its actual model has several extra characteristics.

Firstly, $V_{\text {post }}$ and hence $V_{D}$ are random variables because the initial states of $N$ motors are random, in contrast to CASCADE, where $D$ is independent of initial loads of components. To cope with this, expected values of $V_{\text {post }}$ and $V_{D}$ are used, and are denoted as $\bar{V}_{\text {post }}$ and $\bar{V}_{D}$ respectively. Similar treatment applies to $V_{P}$ and its expected value $\bar{V}_{P}$.

Secondly, actual induction motor cascading stall is a continuous process; i.e., voltage dropping and motor stalling are coupled tightly. It is not likely that the process can be split into discrete stages as shown in Fig. 4, where we assume that voltage dropping makes motors stalled in one stage and motor stalling makes voltage drop in the next stage. The continuous characteristic implies that $\bar{V}_{P}$ should be solved from an implicit equation that takes both voltage dropping and motor stalling into account simultaneously. Subsection IV-C is devoted to this topic.

Thirdly, according to the settings of CASCADE model, $\bar{V}_{\text {post }}$ should be a bus voltage where there are no motors stalled, so that $\bar{V}_{D}$ only reflects the impact of the initial disturbance and is independent of cascading stall process. However, under our settings, when $\bar{V}_{\text {post }}<V_{\max }$, i.e., nontrivial cascades became possible, certain motors will stall immediately if their $V_{c r}>\bar{V}_{\text {post }}$. This difficulty makes necessary an indirect calculation of $\bar{V}_{\text {post }}$ and hence $\bar{V}_{D}$.

Because once cascading stall begins, it continues until the system reaches its post-fault steady state, the expected postfault steady state plays an important role in the analysis. Subsection IV-B gives the method to assess this expected state. We denote the expected post-fault steady state voltage as $\bar{V}_{s}$ and the expected number of motors stalled as $\bar{N}_{s}$. Then given $\bar{V}_{P}, \bar{V}_{\text {post }}$ can be estimated as $\bar{V}_{s}+\bar{N}_{s} \bar{V}_{P}$, and hence

$$
\bar{V}_{D}=V_{\max }-\left(\bar{V}_{s}+\bar{N}_{s} \bar{V}_{P}\right) .
$$

Note that the term $\bar{N}_{s} \bar{V}_{P}$ of (7) approximates the mean of a product as the product of the means.

Similar to (5), after $\bar{V}_{D}$ and $\bar{V}_{P}$ are obtained, the normalized parameters $d$ and $p$ can be calculated as:

$$
\begin{aligned}
& d=\frac{\bar{V}_{D}}{V_{\max }-V_{\min }}, \\
& p=\frac{\bar{V}_{P}}{V_{\max }-V_{\min }} .
\end{aligned}
$$

With estimates for $d$ and $p$, the probability distribution of the number of motors stalled can be calculated with (6). Note that the denominators of (8) and (9) show the influence of the range of stalling voltages $V_{c r}$ on the key cascading parameters.

To put these procedures in perspective, we note that even for a simple system, some assumptions and approximations are needed to carry through the analysis of this paper. While relaxing the assumptions and also generalizing the model have clear value, the path to doing this in an analytic way remains uncertain, while there is a straightforward way to do this by extending the approach of [20] in confirming that the CASCADE model fits simulation results. The insights from analysis of a simplified case can complement the power of simulation and fitting to accommodate more elaborate models that have fewer assumptions.

\section{B. Expected post-fault steady state}

Because the randomness of the system comes from motor's $V_{c r}$ and initial power, the key to obtain $\bar{V}_{s}$ is $\bar{Y}_{M}$, the expected equivalent admittance for motor load.

Denote the random variable for initial motor power as $P_{m 0}$. Then we know from (4) that

$$
\begin{aligned}
& \bar{Y}_{M}(V)=\sum_{i=1}^{N} \bar{Y}_{M i}=N \bar{Y}_{M i} \\
& =N \int_{V_{c r}}\left[\int_{P_{m 0}} Y_{M i} f\left(P_{m 0}\right) d P_{m 0}\right] g\left(V_{c r}\right) d V_{c r},
\end{aligned}
$$

where $f$ and $g$ are the probability density functions for random variables $P_{m 0}$ and $V_{c r}$ respectively.

From the initialization procedure [20], we know that only $P_{m 0}$ influences $C_{i}$, and that $C_{i}$ is proportional to $P_{m 0}$. Therefore the inner integration over $P_{m 0}$ in (10) leads to a result proportional to the expected value of $P_{m 0}$, which is equivalent to assuming that all the motors have equal initial power. This explains the observations in [20] that the distribution of $N_{s}$ is insensitive to $P_{\text {ratio }}$, and that it is a good approximation to assume all initial motor powers equal. Because of this, we assume that $P_{m 0}$ is constant in the following, unless the random $P_{m 0}$ is explicitly discussed.

Taking account of (3) and the assumptions about $V_{c r},(10)$ simplifies to

$$
\begin{aligned}
& \bar{Y}_{M}(V)=N \int_{V_{\min }}^{V_{\max }} \frac{Y_{M i}}{V_{\max }-V_{\min }} d V_{c r} \\
& =N \frac{\int_{V_{\min }}^{V} Y_{M}^{n}\left(V, V_{c r}\right) d V_{c r}+\int_{V}^{V_{\max }} Y_{M}^{s}\left(V_{c r}\right) d V_{c r}}{V_{\max }-V_{\min }} .
\end{aligned}
$$

Here, $Y_{M}^{n}$ and $Y_{M}^{s}$ take the forms in (3), but since all motors are treated as the same, the subscript $i$ is omitted hereafter.

$\bar{V}_{s}$ is the solution of (1) with $G_{M}+j B_{M}$ equaling to $\bar{Y}_{M}(V)$ in (11). However, since the underlying equation for $V$ is too complex to be solved directly, an iteration algorithm similar to that in [20] is adopted. That is, for an estimate of $V$, calculate $\bar{Y}_{M}$ according to (11), then substitute into (1) to get a new $V$. If the error between estimated and new obtained $V$ is small enough, then the obtained $V$ is $\bar{V}_{s}$, otherwise construct a new estimate and iterate again.

Because $V_{c r}$ is uniformly distributed in the interval $\left[V_{\min }, V_{\max }\right]$ and the motors whose $V_{c r}>\bar{V}_{s}$ must be stalled, the expected number of motors stalled at $\bar{V}_{s}$ is

$$
\bar{N}_{s}=N \frac{V_{\max }-\bar{V}_{s}}{V_{\max }-V_{\min }} .
$$

Obviously, $\bar{N}_{s}$ is not likely to be an integer. 


\section{Formula for $\bar{V}_{P}$}

Assume $N_{s}$ motors stalled. For any particular value of $V$, the $V_{c r}$ of $N_{s}$ stalled motors must be greater than $V$. That is, the $V_{c r}$ of the stalled motors must lie in $\left[V, V_{\max }\right]$. Before our assumption of stalling, $V_{c r}$ of these motors was uniformly distributed in $\left[V_{\min }, V_{\max }\right]$. After our assumption of stalling, these $N_{s}$ motors are restricted to the interval $\left[V, V_{\max }\right]$. A random variable uniform on $\left[V_{\min }, V_{\max }\right]$ that is restricted to $\left[V, V_{\max }\right]$ becomes a random variable uniform on $\left[V, V_{\max }\right]$. Therefore $V_{c r}$ of the $N_{s}$ stalled motors is uniformly distributed in $\left[V, V_{\max }\right]$. Similarly, for any particular value of $V$, the $N-N_{s}$ unstalled motors must have $V_{c r}<V$ and hence are uniformly distributed on $\left[V_{\min }, V\right]$. Thus, the expected admittance for motor load at $V$ with $N_{s}$ motors stalled is

$$
\begin{aligned}
\bar{Y}_{M}\left(V, N_{s}\right)= & \left(N-N_{s}\right) \int_{V_{\min }}^{V} \frac{Y_{M}^{n}\left(V, V_{c r}\right)}{V-V_{\min }} d V_{c r} \\
& +N_{s} \int_{V}^{V_{\max }} \frac{Y_{M}^{s}\left(V_{c r}\right)}{V_{\max }-V} d V_{c r} .
\end{aligned}
$$

If we let $N_{s}=N\left(V_{\max }-V\right) /\left(V_{\max }-V_{\min }\right)$, then (13) will be reduced to (11). Hence (13) is a special form of (11), where $N_{s}$ is irrelevant to $V$. It is clear that $\bar{Y}_{M}\left(\bar{V}_{s}, \bar{N}_{s}\right)$ from (13) equals $\bar{Y}_{M}\left(\bar{V}_{s}\right)$ from (11).

Using (13), we can calculate $\bar{Y}_{M}\left(\bar{V}_{s}-\bar{V}_{P}, \bar{N}_{s}+1\right)$, i.e., the expected admittance of motor load when $\bar{V}_{s}$ drops by $\bar{V}_{P}$ and an additional motor beyond $\bar{N}_{s}$ stalls. When $G_{M}+j B_{M}$ in (1) is substituted by $\bar{Y}_{M}\left(\bar{V}_{s}-\bar{V}_{P}, \bar{N}_{s}+1\right)$, the resulting $V$ should be equal to $\bar{V}_{s}-\bar{V}_{P}$ according to the definition of $\bar{V}_{P}$. This relation presents an implicit equation for $\bar{V}_{P}$ once the expected post-fault steady state of the system is obtained.

Alternatively, we can first calculate the admittance increase of motor load at post-fault steady state when $\bar{V}_{s}$ drops by $\bar{V}_{P}$ and an additional motor beyond $\bar{N}_{s}$ stalls, which is

$$
\Delta \bar{Y}_{M}=\bar{Y}_{M}\left(\bar{V}_{s}-\bar{V}_{P}, \bar{N}_{s}+1\right)-\bar{Y}_{M}\left(\bar{V}_{s}, \bar{N}_{s}\right) .
$$

Using linear approximation of (1), we have

$$
\begin{aligned}
& \bar{V}_{P}=-\left.\frac{\partial V}{\partial G_{M}}\right|_{\bar{V}_{s}} \Delta \bar{G}_{M}-\left.\frac{\partial V}{\partial B_{M}}\right|_{\bar{V}_{s}} \Delta \bar{B}_{M} \\
& =\frac{E}{x}\left[\left(G_{Z}+\bar{G}_{M}\right)^{2}+\left(B_{Z}+\bar{B}_{M}-\frac{1}{x}\right)^{2}\right]^{-\frac{3}{2}} \\
& {\left[\left(G_{Z}+\bar{G}_{M}\right) \Delta \bar{G}_{M}+\left(B_{Z}+\bar{B}_{M}-\frac{1}{x}\right) \Delta \bar{B}_{M}\right],}
\end{aligned}
$$

where $\bar{G}_{M}+j \bar{B}_{M}=\bar{Y}_{M}\left(\bar{V}_{s}, \bar{N}_{s}\right)$ and $\Delta \bar{G}_{M}+j \Delta \bar{B}_{M}=$ $\Delta \bar{Y}_{M}{ }^{2}$

Linearization does not change the essence of the implicit equation for $\bar{V}_{P}$ and both linear and nonlinear approaches need an iterative solver, which is designed as:

1) Assess post-fault steady state and obtain $\bar{V}_{s}$ and $\bar{N}_{s}$;

2) Initialize $G_{M}+j B_{M}$ as $\bar{Y}_{M}\left(\bar{V}_{s}, \bar{N}_{s}+1\right)$ and calculate $\bar{V}_{P}$ through the nonlinear equation (1) or the linear approximation equation (14);

3) Update $G_{M}+j B_{M}$ to $\bar{Y}_{M}\left(\bar{V}_{s}-\bar{V}_{P}, \bar{N}_{s}+1\right)$;

\footnotetext{
${ }^{2}$ Because $\bar{V}_{P}$ is a drop in expected $V$, the right hand side of (14) has been multiplied by -1 .
}

4) Calculate a new $\bar{V}_{P}$;

5) If the change in $\bar{V}_{P}$ is small enough, stop the procedure; otherwise, goto step 3 .

\section{A remark about equation (11)}

Equation (11) gives the integration formula for $\bar{Y}_{M}$, which can be approximated using the midpoint rule as

$$
\begin{aligned}
\bar{Y}_{M}(V) & \approx \frac{N}{V_{\max }-V_{\min }} \sum_{i=1}^{N} Y_{M i}\left(V, V_{c r i}\right) \frac{V_{\max }-V_{\min }}{N} \\
& =\sum_{i=1}^{N} Y_{M i}\left(V, V_{c r i}\right)
\end{aligned}
$$

where the integration interval is split into $N$ segments and

$$
V_{c r i}=V_{\min }+\left(V_{\max }-V_{\min }\right) \frac{i-0.5}{N}, \quad i=1,2, \ldots, N \text {. }
$$

Equation (15) clearly shows that $\bar{Y}_{M}$ can be approximated by the sum of $N$ motors' admittances, where the $N$ motors have equal initial power and their $V_{c r}$ forms an arithmetical series as shown in (16). Furthermore, since the assumed distribution of motor initial power does not change $\bar{Y}_{M}$, the above approximation holds even when the randomness of motor initial power is considered. This observation can have some applications. In particular, it explains the reason why the number of motors stalled and the load bus voltage at system post-fault steady state calculated from a typical sample in [20], which is produced by the method above, could provide insightful information about induction motor cascading stall.

\section{E. $d+N p$ as an indicator for system stress}

$\bar{V}_{D}$ and $\bar{V}_{P}$ describe the expected voltage drop beyond $V_{\max }$ due to the initial disturbance and the expected voltage drop when one extra motor stalled respectively. They can be combined into one index: $\bar{V}_{D}+N \bar{V}_{P}$. If this index approaches $V_{\max }-V_{\min }$, the system is highly stressed and there will be a high risk of massive cascading stall that has the expected number of stalled induction motors comparable to $N$.

To reach this conclusion, we begin with an informal explanation of two special cases and then discuss the general case.

1) $\bar{V}_{D}$ approaches $V_{\max }-V_{\min }$. Obviously, in this case, the initial disturbance is so strong that many motors (in number comparable to $N$ ) will be stalled at once.

2) $\bar{V}_{P}$ approaches $\left(V_{\max }-V_{\min }\right) / N$. In this case, because $V_{c r}$ of each of the $N$ motors is uniformly distributed in the interval $\left[V_{\min }, V_{\max }\right]$, one extra motor is expected to stall once the voltage drops by $\left(V_{\max }-V_{\min }\right) / N$, which is also about $\bar{V}_{P}$ in this case. Therefore, each motor stall is expected to cause another subsequent motor stall and hence the cascading stall process can be propagated to massive motor stall.

3) $\bar{V}_{D}+N \bar{V}_{P}$ approaches $V_{\max }-V_{\min }$. Considering the two special cases and the assumption about $V_{c r}$, no matter how small $\bar{V}_{D}$ is, the cascading stall is expected to propagate similarly to case 2 . 


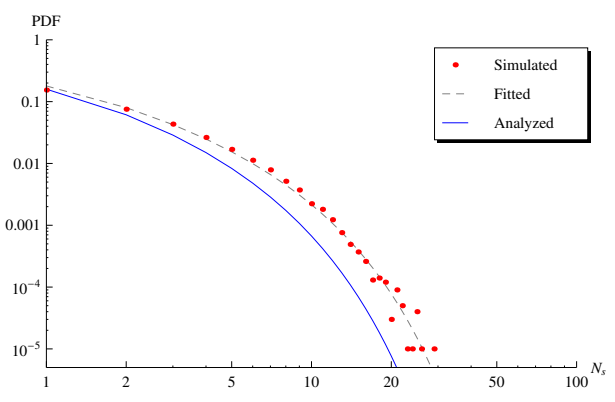

Fig. 5. Distribution of the number of motors stalled $N_{s}$ at base case

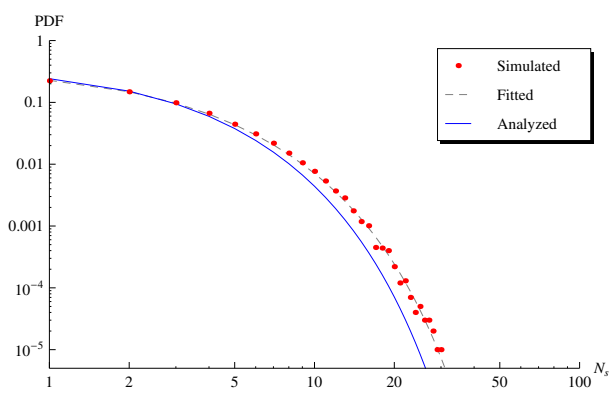

Fig. 6. Distribution of the number of motors stalled $N_{s}$ at $\lambda=1.02$

With the normalization (8) and (9), the index $\bar{V}_{D}+N \bar{V}_{P}$ becomes $d+N p$. $d+N p$ indicates system stress; once $d+$ $N p$ is close to 1.0 , there will be a significant probability of large numbers of motors stalled. This explains the observations about $d+N p$ in [20].

More formally, we note from [19] that $d+n p \geq 1$ is the condition of the CASCADE model allowing the possibility of all the motors to stall at some point in the cascade, which is called "saturation."

\section{CASE STUdies}

\section{A. Method performance and impact of system stress}

The system parameters at base case and the induction motor parameters are same as those in [20]. That is, $E=1.029$, $x_{n}=0.1, x_{p}=0.2, P_{L 0}+j Q_{L 0}=1.0+j 0.4, N=100$, $M_{\text {ratio }}=0.6, V_{\max }=0.92, V_{\min }=0.5, P_{\text {ratio }}=10, r_{1}=$ $0.0456, x_{1}=0.295, r_{2}=0.02, x_{2}=0.12, r_{m}=0.35$, $x_{m}=3.5, \alpha=0.15$ and $p=2.0$, where $x_{n}$ and $x_{p}$ are prefault and post-fault transmission line reactance respectively, and $M_{\text {ratio }}$ is the ratio of motor load to total load. The number of samples $N_{\text {sample }}$ is $10^{5}$.

Fig. 5 compares the distributions of $N_{s}$ obtained from the simulation, fitting and analysis methods at base case. The fitting method, which finds the best values of $d$ and $p$ for which CASCADE best fit the simulated results, is described in more detail in [20]. For these results, the simulated result of the probability of no motor stalled $P_{0}$ is $0.6483, \bar{V}_{s}$ and $\bar{N}_{s}$ are 0.9176 and 0.5702 respectively, and the analyzed $d$ and $p$ are 0.003346 and 0.004132 respectively. It can be seen that the simulated and fitted distribution match quite well and the analyzed distribution has a certain discrepancy from them. Even though, the proposed analysis method can be regarded

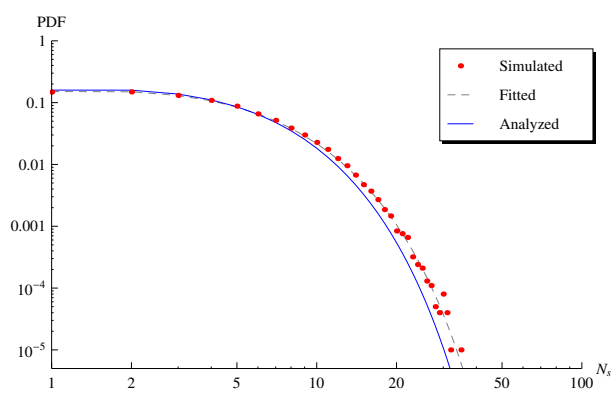

Fig. 7. Distribution of the number of motors stalled $N_{s}$ at $\lambda=1.05$

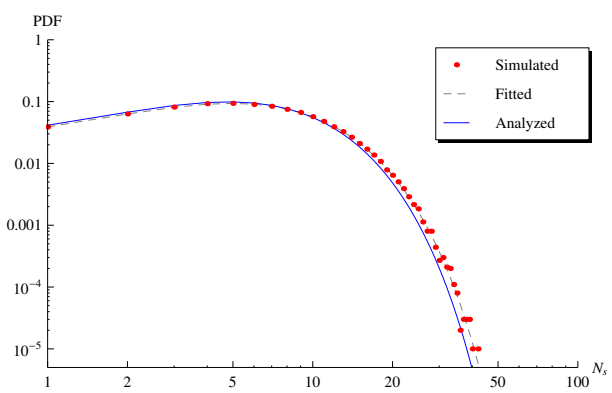

Fig. 8. Distribution of the number of motors stalled $N_{s}$ at $\lambda=1.10$

as quite successful, since it achieves this result through pure analysis based on the CASCADE model.

We examine the effect of changing the initial load power $P_{L 0}+j Q_{L 0}$ by scaling it by a factor $\lambda$. The discrepancy reduces as loading factor $\lambda$ increases, as shown in Figs. 6 and 7 , where the simulated results for $P_{0}$ are 0.3144 and 0.1023 respectively. As we can see, $2 \%$ load increase from the base case gives a good result while a $5 \%$ increase is even better. It is now clear that the proposed analysis method can capture some essential characteristics of induction motor cascading stall. Indeed, Fig. 8 gives the result when load increases $10 \%$, where the simulated result for $P_{0}$ is 0.01417 . Overall, it can be seen that the analyzed distribution matches the simulated and fitted results quite well. Our analytic method achieves better performance under small $P_{0}$ or stressed system conditions.

\section{B. Impact of several variables on $d$ and $p$}

To study the impact of the loading factor $\lambda$ on $d$ and $p, 18$ cases are generated by varying $\lambda$ from 0.96 to 1.25 with step 0.01 when $\lambda \leq 1.10$ and step 0.05 when $\lambda>1.10$. It is found that 16 cases with $\lambda \geq 0.98$ can be fitted with the CASCADE model using the method of [20]. The two failed cases have $P_{0}$ greater than 0.998 so that cascading stalls in these conditions are too rare. It is found that 14 cases with $\lambda \geq 1.00$ can be analyzed with the method of this paper. The four failed cases have $\bar{V}_{s}>V_{\max }$, which means that no motor is expected to be stalled. Figs. 9 and 10 compare $d$ and $p$ obtained from the fitting and analysis methods respectively.

Fig. 9 shows that the fitted and analyzed $d$ match well and that the analyzed $d$ has good linear relationship with $\lambda$ as shown by the fitted straight line. Since $P_{0}=(1-d)^{N}$ according to (6), then $\log P_{0}=N \log (1-d)$ and hence $\Delta\left(\log P_{0}\right)=-N \Delta d /(1-d)$. Because $\Delta d$ is approximately 


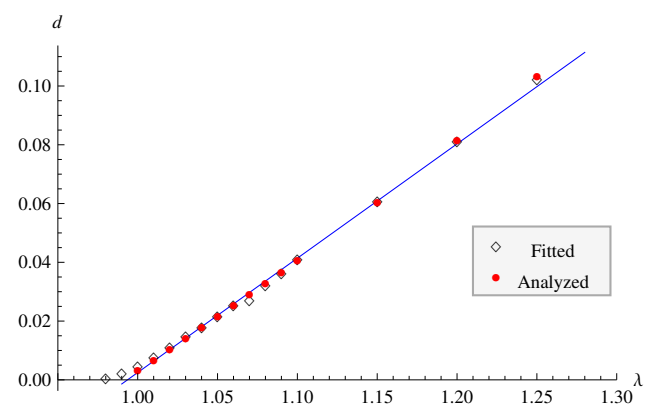

Fig. 9. Comparison of fitted and analyzed $d$ when $\lambda$ changes

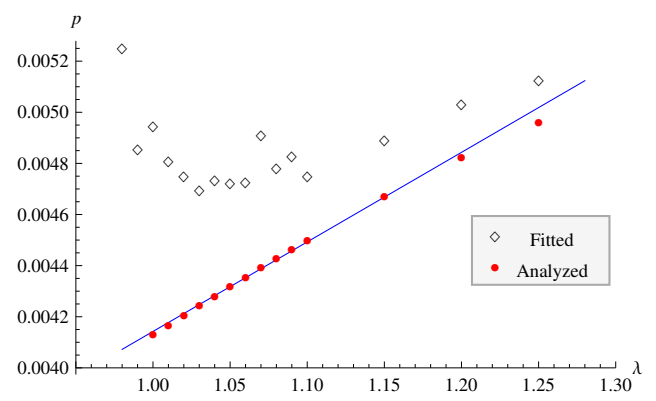

Fig. 10. Comparison of fitted and analyzed $p$ when $\lambda$ changes

proportional to $\Delta \lambda$ and $d$ is basically a small value, we can explain the relationship between $\log P_{0}$ and $\lambda$ in [20].

Fig. 10 shows that analyzed $p$ also has good linear relationship with $\lambda$ as indicated by the fitted straight line. However, fitted $p$ is $3 \%-19 \%$ larger than analyzed $p$ and its pattern is not obvious. Because the analyzed $d$ matches well with fitted $d$ and its derivation takes advantage of analyzed $p$, the analyzed $p$ seems to be accurate. On the other hand, the fitting method from [20] fits the logarithm of (6) to the logarithm of probabilities and hence gives more weight to the tail of the $N_{s}$ distribution. It seems that a bigger $p$ in these cases can improve the fitting performance at the tail of distribution, exactly where the largest errors of the analyzed results are as we can see from Figs. 5 to 8.

The above impact of $\lambda$ on $d$ and $p$ is quite typical. Studies reveal that $P_{L 0}, Q_{L 0}$ and $M_{\text {ratio }}$ also have good linear relationships with the analyzed $d$ or $p$. Due to space limitations, the detailed comparisons are not described here. Table I summarizes the sensitivities, i.e., the slopes of fitted straight line, of analyzed $d$ and $p$ to $\lambda, P_{L 0}, Q_{L 0}$ and $M_{\text {ratio }}$, from which we have following observations:

1) The values in the $\lambda$ column approximately equal the values in the $P_{L 0}$ column plus 0.4 times the values in the $Q_{L 0}$ column, where 0.4 comes from the fact that at base case, $Q_{L 0}=0.4 P_{L 0}$. This implies $P_{L 0}$ and $Q_{L 0}$

TABLE I

SENSitivities of ANALYZED $d$ AND $p$ To $\lambda, P_{L 0}, Q_{L 0}$ AND $M_{\text {ratio }}$

\begin{tabular}{ccccc} 
& $\lambda$ & $P_{L 0}$ & $Q_{L 0}$ & $M_{\text {ratio }}$ \\
\hline sensitivity of $d$ & 0.3891 & 0.1737 & 0.5264 & 0.09565 \\
sensitivity of $p$ & 0.003504 & 0.004495 & -0.001799 & 0.009775 \\
\hline
\end{tabular}

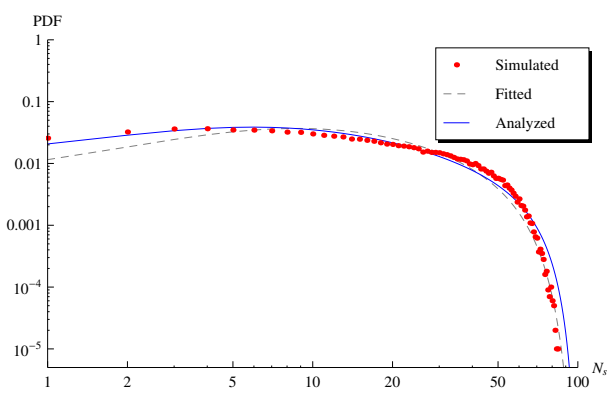

Fig. 11. Distribution of number of motors stalled $N_{s}$ when $M_{\text {ratio }}=1.0$

roughly influence $d$ or $p$ independently.

2) $d$ has the biggest sensitivity to $Q_{L 0}$, which means increasing load compensation can efficiently prevent cascading stall from happening. However, the negative sensitivity of $p$ to $Q_{L 0}$ indicates that increasing compensation makes cascading stall easier to propagate and hence makes large cascading stalls relatively more likely than small cascading stalls. Our analysis reveals this non-obvious tradeoff between the frequency and severity of cascading.

3) $d$ has the smallest sensitivity to $M_{\text {ratio }}$, which means that increasing the percentage of motor load will not decrease $P_{0}$ significantly. However the large sensitivity of $p$ to $M_{\text {ratio }}$ indicates that increasing the percentage of motors will incur more frequent large cascading stalls.

\section{Highly stressed cases}

When system is highly stressed, which can be indicated by $d+N p$ close to 1.0 , there will be a high risk of massive cascading stall and the distribution could be hard to fit with the CASCADE model [20].

Fig. 11 shows the distribution of $N_{s}$ when $M_{\text {ratio }}=1.0$, whose simulated $P_{0}=0.01428, \bar{V}_{s}=0.8255$ and $\bar{N}_{s}=$ 22.51. The analyzed $d$ and $p$ are 0.04488 and 0.008006 respectively and hence $d+N p=0.8455$. As we can see, both analyzed and fitted distributions match the simulated distribution well. Compared with Fig. 8, whose simulated $P_{0}$ is 0.01417 , analyzed $d$ and $p$ are 0.04078 and 0.004501 respectively and hence $d+N p=0.4909$, Fig. 11 has a similar $P_{0}$ but an extended distribution and a higher probability of large number of motors stalled. ${ }^{3}$

Fig. 12 gives the distribution of $N_{s}$ when $V_{\max }=0.85$ and $P_{L 0}+j Q_{L 0}=1.697+j 0.4$, whose simulated $P_{0}=0.5644$, $\bar{V}_{s}=0.6706, \bar{N}_{s}=51.26$. This case is special because with typical sample, the system has no motor stalled and $V_{s}=$

\footnotetext{
${ }^{3}$ A careful inspection of the distributions of $N_{s}$ in Fig. 11 shows that for two parts of the PDF, the simulated data have a lower probability density than the analytical model, which contrasts with Figs. 5 to 8, where the simulated data almost consistently have a higher probability density. The reason is that in the stressed system of Fig. 11, massive cascading stall becomes more likely and hence $\bar{V}_{P}$ could be overestimated, while in Figs. 5 to 8 where the system is not stressed much $(d+N p<0.5)$, the cascading stalls are not fully developed and hence $\bar{V}_{P}$ is likely to be underestimated. In our analysis method, it is implicitly assumed that each extra stalled motor causes a mean voltage drop of $\bar{V}_{P}$; i.e., the mean voltage drops linearly with the number of motors stalled. However, the underlying problem is nonlinear, and the effects of our linear approximation can appear in different ways depending on system conditions.
} 


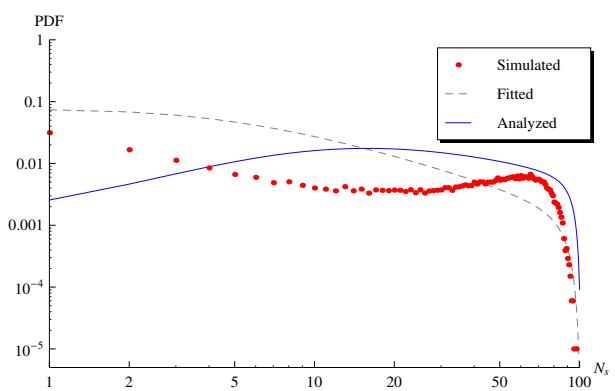

Fig. 12. Distribution of number of motors stalled $N_{s}$ when $V_{c r}=0.85$ and $P_{L 0}+j Q_{L 0}=1.697+j 0.4$

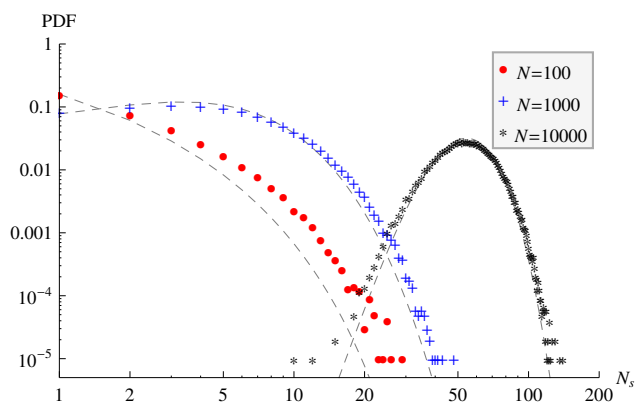

Fig. 13. Distribution of number of motors stalled $N_{s}$ with different $N$ at base case.

0.8483 , while a 0.001 increase of $P_{L 0}$ will cause 49 motors stalled and $V_{s}=0.6772$. The analyzed $d$ and $p$ are 0.06787 and 0.008676 respectively, and hence $d+N p=0.9355$. Because $d+N p$ is close to 1.0 , the analyzed distribution cannot match well the simulated distribution but can predict the significant probability of a large numbers of motors stalled.

\section{Impact of number of motors $N$ on $N_{s}$ distribution}

So far, all cases have $N=100$. Now let's increase $N$ and see how the distribution of $N_{s}$ changes. Figs. 13-15 compare $N_{s}$ distributions of three cases with different $N$, where the dashed lines correspond to analyzed $N_{s}$ distributions. The three cases are the base case, the base case except that $M_{\text {ratio }}=0.8$, and the base case except that $V_{\max }=0.85$ and $P_{L 0}+j Q_{L 0}=1.697+j 0.4$. Each case is simulated and analyzed with $N$ being set to 100,1000 and 10000 respectively, except for the case with $V_{\max }=0.85$ and $N=10000$, whose required simulation time is prohibitively large. Table II summarizes five key parameters of each case with different $N$.

From Figs. 13-15 and table II, we have the following observations and conclusions:

1) $\bar{V}_{s}$ is independent of $N$ and $\bar{N}_{s}$ is proportional to $N$.

2) Approximately, $p$ is inversely proportional to $N$ and $d$ is independent of $N$, and hence $d+N p$ is also independent of $N$. Because $P_{0}=(1-d)^{N}, P_{0}$ decreases exponentially when $N$ increases.

3) Fig. 13 shows that when $d+N p$ is small, the probability of a large number (compared with $N$ ) of motors stalled is also small. However, the form of the $N_{s}$ distribution varies with $N$. For $N=100, \bar{N}_{s}$ is small and the

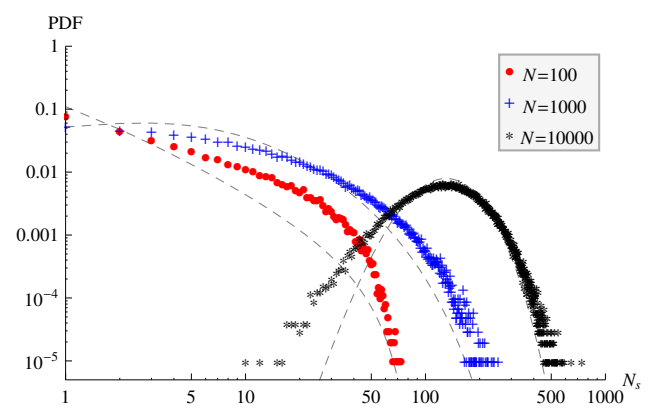

Fig. 14. Distribution of number of motors stalled $N_{s}$ with different $N$ when $M_{\text {ratio }}=0.8$

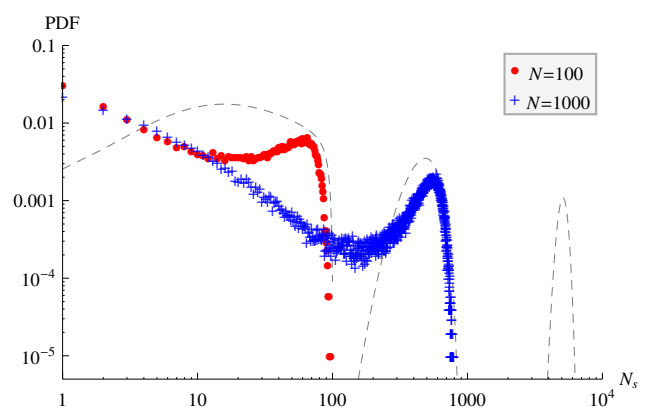

Fig. 15. Distribution of number of motors stalled $N_{s}$ with different $N$ when $V_{\max }=0.85$ and $P_{L 0}+j Q_{L 0}=1.697+j 0.4$. Because of prohibitive computation involved, simulation results of $N=10000$ are not shown.

$N_{s}$ distribution has a typical heavy tailed shape. For $N=10000, \bar{N}_{s}$ is proportionally larger and the $N_{s}$ distribution is peaked.

4) Fig. 14 has essentially same characteristic as Fig. 13, although the system has a bigger $d+N p$ and more motors will stall. The main difference is that the diversity of the $N_{s}$ distribution increases. For example, when $N=10000$, about $0.2 \%-5 \%$ motors stall in Fig. 14, whereas about $0.15 \%-1.1 \%$ motors stall in Fig. 13 .

5) Fig. 15 shows that when $d+N p$ exceeds 0.93 , there

TABLE II

IMPACT OF NUMBER OF MOTORS $N$ ON KEY PARAMETERS

\begin{tabular}{lllll} 
& & $N=100$ & $N=1000$ & $N=10000$ \\
\hline \multirow{2}{*}{ base } & $\bar{V}_{s}$ & 0.9176 & 0.9176 & 0.9176 \\
& $\bar{N}_{s}$ & 0.5702 & 5.702 & 57.02 \\
& $d$ & 0.003346 & 0.003351 & 0.003351 \\
& $p$ & 0.004132 & 0.0004123 & 0.00004123 \\
& $d+N p$ & 0.4165 & 0.4157 & 0.4156 \\
\hline \multirow{3}{*}{$M_{\text {ratio }}$} & $\bar{V}_{s}$ & 0.9135 & 0.9135 & 0.9135 \\
& $\bar{N}_{s}$ & 1.544 & 15.44 & 154.4 \\
& $d$ & 0.003335 & 0.003388 & 0.003391 \\
& $p$ & 0.007840 & 0.0007806 & 0.00007804 \\
& $d+N p$ & 0.7873 & 0.7840 & 0.7838 \\
\hline \multirow{2}{*}{$\begin{array}{l}\text { max } \\
=0.85\end{array}$} & $\bar{V}_{s}$ & 0.6706 & 0.6706 & 0.6706 \\
& $\bar{N}_{s}$ & 51.26 & 512.6 & 5126. \\
& $p$ & 0.06787 & 0.06979 & 0.06982 \\
& $d+N p$ & 0.008676 & 0.0008638 & 0.00008638 \\
& & 0.9355 & 0.9336 & 0.9336 \\
\hline
\end{tabular}


is a significant probability of a large number of motors stalled. Because the high probability of large numbers of motors stalled coexists with a high simulated $P_{0}$, which is 0.5644 for $N=100$ and 0.2891 for $N=1000$, special attention should be paid to such cases.

6) When $d+N p$ is not large, the analyzed $N_{s}$ distribution matches well with simulated $N_{s}$ distribution. When $d+N p$ is close to 1.0 , the match is not close, but one can correctly predict the risk that a large numbers of motors stall. It should be noted that the proposed analysis method has a negligible computation time compared with simulation.

\section{CONCLUSION}

We use analytic methods to predict the probability distribution of the number of motors stalled in a single line power system with a load with many induction motors. We derive CASCADE model parameters $d$ and $p$ describing the initial disturbance and average amount of propagation from the system parameters, and then obtain the probability distribution of numbers of motors stalled using the CASCADE model formula. Case studies comparing the analytic approach with simulations show that when system is moderately stressed, the method works well, while when system is highly stressed, it can evaluate the risk of large numbers of motors stalled. This demonstrates the use of CASCADE and its parameters $d$ and $p$ to quantitatively describe cascading stall. The analysis is more insightful than simulation and also orders of magnitude faster.

The analysis quantifies relationships so that we can begin to understand how to make changes in the system parameters and $d$ and $p$ to mitigate large cascading stalls. Our case studies show that the load level, initial real and reactive load, and the ratio of motor load in total load all have good linear relationships with $d$ or $p$. Hence the system changes rapidly from unlikely cascading stall to frequent cascading stall. Static reactive power compensation can help to prevent the initiation of cascading stall but the resulting fewer cascades will propagate more easily. $d+N p$ is a useful indicator for the system, where $N$ is the number of motors. If $d+N p$ is near 1 , the probability of a large numbers of motors stalled is high and its distribution is largely independent of $N$.

Analytic calculations on simple systems often reveal relationships and insights that can underpin engineering solutions, and it is reasonable to ask how our methods could be applied more generally. We note that [20] discusses the prospects for generalization to more elaborate systems models, and in particular [20, section VII] suggests that the CASCADE parameters $d$ and $p$ could be estimated from measurements at a substation bus.

\section{REFERENCES}

[1] W. D. Humpage, K. E. Durrani, and V. F. Carvalho, "Dynamic-response analysis of interconnected synchronous-asynchronous-machine groups," Proc. IEE, vol.116, no.12, Dec. 1969.

[2] R. Ueda, S. Takata, "Effects of induction machine load on power system," IEEE Trans. Power Apparatus and Systems, vol.100, no.5, pp.2555-2562, May 1981.

[3] C. W. Taylor, Power system voltage stability, McGraw-Hill, NY, 1994.
[4] T. Van Cutsem, C. Vournas, Voltage stability of electric power systems, Kluwer Academic Publishers, Boston, 1998.

[5] J. A. Diaz de Leon II, C. W. Taylor, "Understanding and solving shortterm voltage stability problems," in Proc. IEEE Power Eng. Soc. Summer Meeting, 2002, vol.2, pp.745-752.

[6] NERC Transmission issues and System protection and control subcommittees, "Fault-induced delayed voltage recovery," June 2009.

[7] M.M. Abdel Hakim, G.J. Berg, "Dynamic single-unit representation of induction motor groups," IEEE Trans. Power App. Syst., vol.95, no.1, pp.155-165, Jan. 1976.

[8] W.W. Price, K.A. Wirgau, A. Murdoch, J.V. Mitsche, E. Vaahedi, M.A. El-Kady, "Load modeling for power flow and transient stability computer studies," IEEE Trans. Power Syst., vol.3, no.1, pp.180-187, Feb. 1988.

[9] IEEE Task force on load representation for dynamic performance, "Standard load models for power flow and dynamic performance simulation," IEEE Trans. Power Syst., vol.10, no.3, pp.1302-1313, Aug. 1995.

[10] L. Pereira, D. Kosterev, P. Mackin, D. Davies, J. Undrill, W. Zhu, "An interim dynamic induction motor model for stability studies in the WSCC," IEEE Trans. Power Syst., vol.17, no.4, pp.1108-1115, Nov 2002.

[11] D. Kosterev, A. Meklin, J. Undrill, B. Lesieutre, W. Price, D. Chassin, R. Bravo, S. Yang, "Load modeling in power system studies: WECC progress update," IEEE PES General Meeting, Pittsburgh, PA USA, July 20-24, 2008.

[12] B. Lesieutre, R. Bravo, R. Yinger, D. Chassin, H. Huang, N. Lu, I. Hiskens, and G. Venkataramanan, Load modeling transmission research, Final Report, California Institute for Energy and Environment, March 2010. Online: http://www.uc-ciee.org/downloads/LM_Final_Report.pdf.

[13] C.D. Vournas, G.A. Manos, "Modeling of stalling motors during voltage stability studies," IEEE Trans. Power syst., vol.13, no.3, pp.775-781, Aug. 1998.

[14] A. M. Stankovic, B. C. Lesieutre, "Parametric variations in dynamic models of induction machine clusters," IEEE Trans. Power Syst., vol.12, no.4, pp.1549-1554, Nov. 1997.

[15] Y. Mishra, Z.Y. Dong, J. Ma, D.J. Hill, "Induction motor load impact on power system eigenvalue sensitivity analysis," IET Gener. Transm. Distrib., vol.3, no.7, pp.690-700, Jul. 2009.

[16] B.A. Carreras, D.E. Newman, I. Dobson, A.B. Poole, "Evidence for selforganized criticality in a time series of electric power system blackouts," IEEE Trans. Circuits Syst. I, vol.51, no.9, pp.1733-1740, Sept. 2004.

[17] I. Dobson, B. A. Carreras, V. E. Lynch, D. E. Newman, "Complex systems analysis of series of blackouts: cascading failure, critical points, and self-organization," Chaos, vol.17, 026103, 2007.

[18] IEEE PES CAMS Task Force on Understanding, Prediction, Mitigation and Restoration of Cascading Failures, "Initial review of methods for cascading failure analysis in electric power transmission systems," IEEE PES General Meeting, Pittsburgh, PA USA, July 20-24, 2008.

[19] I. Dobson, B. A. Carreras, D. E. Newman, "A loading-dependent model of probabilistic cascading failure," Probability in the Engineering and Informational Sciences, vol.19, pp.15-32, 2005.

[20] H. Wu, I. Dobson, "Cascading stall of many induction motors in a simple system," IEEE Trans. Power Syst., vol. 27, no. 4, pp. 2116-2126, Nov. 2012.

[21] Xi-Fan Wang, Yonghua Song, Malcolm Irving, Modern power systems analysis, Springer-Verlag, New York, 2008.

[22] I. Dobson, B.A. Carreras, D.E. Newman, "A probabilistic loadingdependent model of cascading failure and possible implications for blackouts," 36th Hawaii Intl. Conf. System Sciences, Hawaii, January 2003.

[23] C. Lefèvre, "On the outcome of a cascading failure model," Probability in the Engineering and Informational Sciences, vol. 20, pp. 413-427, 2006.

Hao Wu (M'10) received Ph.D. degree in electrical engineering from Zhejiang University, Hangzhou, Zhejiang, China. He joined Zhejiang University in 2002, visited Hong Kong Polytechnic University in 2003 2004 and University of Wisconsin-Madison in $2009-2011$.

Ian Dobson (F'06) received the Ph.D. in electrical engineering from Cornell University, and is Sandbulte professor in electrical and computer engineering at Iowa State University. 